
\title{
ECOLOGY OF MOSSES
}

\author{
Brent D. Mishler ${ }^{1}$ and Melvin J. Oliver ${ }^{2}$ \\ ${ }^{1}$ Department of Integrative Biology, University of California, Berkeley, \\ CA 94720-2465, USA \\ 2 Plant Genetics Research Group, USDA-ARS University of Missouri, Columbia, MO \\ 65211, USA
}

\begin{abstract}
Physcomitrella patens is an important model system for studies of genetics and physiology, and with its newly sequenced genome, it is perfectly placed phylogenetically to serve as a point of comparison for angiosperms. This chapter addresses three main questions: (1) How typical of a moss is P. patens? It is rather atypical, given the reasons that it was selected as a model system, such as its rapid life cycle and reduced morphology, yet it is representative in many ways. (2) Where does it belong in the phylogenetic history of land plants? The mosses are monophyletic, and share a common ancestor with hornworts and tracheophytes, with $P$. patens nesting within the 'true mosses'. (3) What are the special attributes of moss ecology and evolution that can lend special interest to the study of $P$. patens? When comparing P. patens with tracheophytes, it is important to understand both its similarities and differences with its larger cousins - we discuss how many processes influencing their physiology, ecology and evolutionary diversification seem to be quite different from the tracheophytes.
\end{abstract}

Keywords: desiccation tolerance; ecology; evolution; mosses; phylogeny; Physcomitrella

\subsection{Introduction to Physcomitrella}

The moss Physcomitrella patens (Hedw.) Bruch \& Schimp. (sometimes called Aphanorrhegma patens) is becoming widely recognized as an experimental model of choice not only for basic molecular, cytological and developmental questions in plant biology, but also as a key link in understanding questions 
in plant phylogeny, most recently those related to genome evolution. P. patens is well placed phylogenetically to provide important comparisons with the flowering plants. In terms of evolutionary distance, P. patens is to the flowering plants as Drosophila melanogaster is to humans! The completion of the sequencing of the P. patens genome (Rensing et al., 2008) allows bioinformatic and functional genomic comparisons to be carried out in plants, just as the Mus musculus, Fugu rubripes, D. melanogaster and Caenorhabditis elegans genomes have informed animal biology.

An increasing number of laboratories worldwide are studying this moss. P. patens has been developed into an important model for investigating gene function (Reski, 1998, 1999; Cove, 2000). A large reason for its growing popularity lies in the fact that it can be cultured rapidly on a simple medium containing only inorganic salts, its life cycle can be completed in culture in about 3 months, and since the dominant phase of the life cycle, the gametophyte, is haploid, mutant phenotypes can be observed directly in progeny. The simple morphology allows direct observation of intracellular processes involved in morphogenesis. Sexual crossing, somatic hybridization and the generation of transgenic plants are now routine.

The utility of $P$. patens is reinforced, since in contrast to vascular plants, transforming DNA containing a genomic sequence targets the corresponding sequence in the genome at very high frequency (see Chapter 4). The high frequency of gene targeting allows disruption and/or replacement of genes as efficiently as Saccharomyces cerevisiae and orders of magnitude more efficiently than any other plant system. P. patens is thus the first land plant that allows easy targeting of genes for replacement and elimination.

The increasing popularity of $P$. patens as a study system has focused new attention on the mosses, a diverse yet relatively unknown group of plants. To use this model properly to study general questions in plant biology, it is important to address several questions: How typical of a moss is P. patens? Where does it belong in the phylogenetic history of land plants? What are the special attributes of moss ecology and evolution that can lend special interest to the study of $P$. patens? Such questions will be addressed in the following sections of this chapter.

\subsection{The position of $P$. patens on the Tree of Life}

P. patens is a green plant, thus belonging to one of the six major clades of crown eukaryotes (the other five are: animals, fungi, red plants, stramenopiles and alveolates; Mishler, 2000). More specifically, P. patens is a land plant, and even more specifically, it is a moss. Our understanding of its precise position in this deeply nested phylogenetic hierarchy has been greatly enhanced over the last 15 years. Results to date suggest that the green plants appear to be composed of two major lineages and a residuum of unicellular prasinophytes (see Figure 1.1. For an elaborate tree shown in hyperbolic space, see 


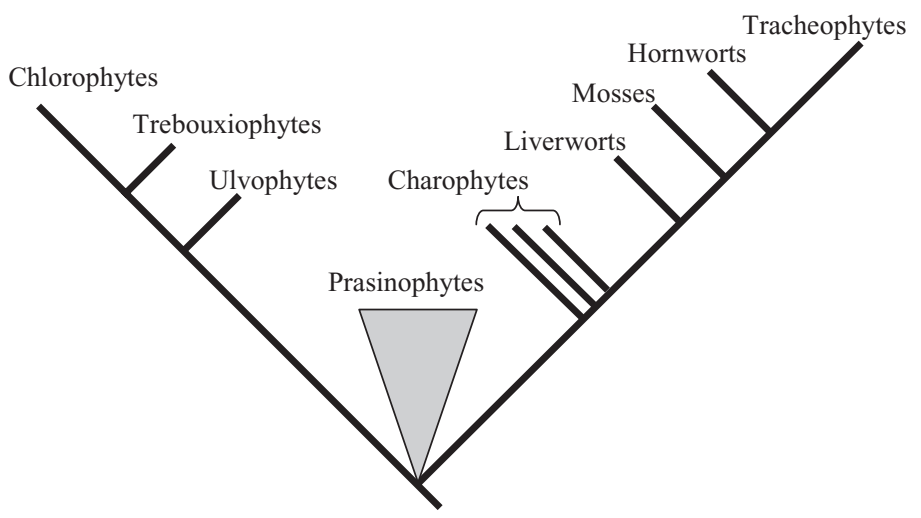

Figure 1.1 A summation of the currently hypothesized cladistic relationships of green plants. Chlamydomonas and Volvox are members of the farthest left clade, while flowering plants are nested well up in the farthest right clade. The marine green algae are in the Ulvophytes. The prasinophytes are a residual group of unicells whose relationships are not yet clear. The charophytes are a paraphyletic assemblage of land plant relatives that include Chara, Coleochaete and Spirogyra. P. patens is a moss.

http://ucjeps .berkeley.edu/TreeofLife/hyperbolic.php). One of these major lineages contains the bulk of the classical green algae (Chlorophyceae, Trebouxiophyceae and Ulvophyceae), including all the marine forms. The other contains the embryophytes (land plants) plus some of the former green algae, such as Spirogyra and Chara. Within the embryophytes, there are four major lineages, that is, liverworts, hornworts, mosses and the tracheophytes (vascular plants); the first three are collectively called bryophytes.

The relationships of these four major groups to one another are not fully agreed upon. Virtually all of the recently published studies agree that the traditional group 'bryophytes' is paraphyletic (i.e. this group does not contain all the descendants of its common ancestor since the tracheophytes are left out), and that the basal split in the embryophytes is between the liverworts and the rest. The relative positions of hornworts and mosses are more controversial, but most recent studies suggest that the mosses diverged first, and thus the hornworts and tracheophytes are sister groups (Kelch et al., 2004; Qiu et al., 2006). Considerable effort is now being expended to better resolve these relationships and other uncertain nodes in the land plant phylogeny (i.e. the Green Tree of Life Project funded by the National Science Foundation Assembling the Tree of Life (AToL) program; see http://ucjeps. berkeley.edu/TreeofLife/).

Within the tracheophytes, the lycophytes (club mosses) are sister to all other living tracheophytes, and within the tracheophytes, ferns and their allies are sister to the seed plants (Pryer et al., 2001). Within the seed plants, morphological and molecular data currently provide conflicting topologies for the five extant lineages (cycads, Ginkgo, conifers, Gnetales and angiosperms). 
Further work is underway to resolve relationships among lineages of seed plants, also funded by the NSF AToL program (see http://www.huh. harvard.edu/research/mathews-lab/atolHtmlsite/ and http:// www. flmnh.ufl.edu/angiospermATOL/).

The rapidly developing green plant phylogeny described above can serve as a framework for evolutionary interpretations (Mishler, 2000). For example, it appears to support reasonably well that multicellularity arose at least twice in the green plants. The diversification of life-history strategies is becoming clearer; from a primitively haploid-dominant life cycle, both alternation of generations and diploid-dominant life cycles arose at least twice. The habitat transition in the movement of plants to land was from fresh water, not from salt water. Within the land plants, several morphological transformations can be reasonably postulated at present, such as the multiple elaborations of the gametophyte and sporophyte in all four main lineages, the radiation of types of conducting cells, and the single origin of a branched, multisporangiate sporophyte from unbranched, unisporangiate ones in the tracheophytes. We can also begin to understand genome evolution in the same manner, once major gaps in the phylogenetic coverage among green plants are filled. $P$. patens is placed in a strategic position in the phylogeny of green plants to provide insight into such topics.

\subsection{Relationships within the mosses}

Phylogenetic relationships are becoming well supported within the moss clade (see Figure 1.2; Newton et al., 2000; Cox et al., 2004). The first major phylogenetic split within the mosses is Sphagnum versus the remainder of mosses. The enigmatic Takakia, only recently discovered to be a moss rather than a liverwort (Smith and Davison, 1993), is still of uncertain placement, either as sister to Sphagnum, or diverging earliest among extant mosses. The next major split involves the divergence of the Andreales from the main line, followed by the Polytrichales. The 'true mosses' (see arrow 1 in Figure 1.2), that is, those mosses with articulated and hygroscopic peristomes are the most diverse group in terms of number of species. Within this group, the largest clade (see arrow 2 in Figure 1.2) is the group with alternate peristomial formulae (i.e. Timmia and the Haplolepideae, plus the diplolepideous-alternate mosses including the pleurocarps).

The sister to this group is the Funariales, where P. patens is located (shown with an asterisk in Figure 1.2). The Funariales have a diplolepideous-opposite peristomial formula (Goffinet et al., 1999), and a generalized leaf form with broad leaves and rectangular leaf cells. Interestingly, this leaf form is characteristic of juvenile leaves in the heteroblastic series of all the mosses (Mishler, 1988), perhaps indicating that it represents the primitive form from which the many diverse types of moss leaf morphology have evolved. Unlike many mosses, species within the Funariales often have an annual life history, with 


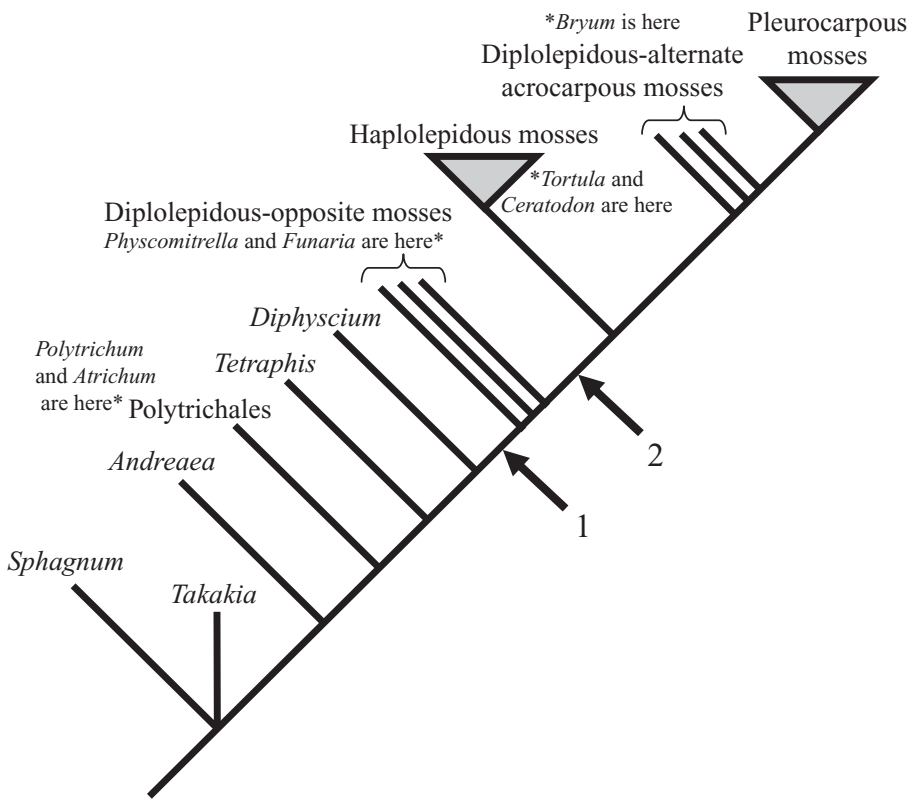

Figure 1.2 A simplified phylogeny of the mosses. Arrow 1 shows the mosses with a true hygroscopic peristome. The diplolepideous-opposite peristomial arrangement is primitive; arrow 2 shows where the alternate peristomial formula evolves, uniting the majority of moss species. See the text for further explanation.

P. patens showing an extreme version. Many species within the Funariales have a truncated sporophyte development, often lacking a peristome and operculum entirely as in P. patens.

This topology can be used to study evolutionary transitions within the mosses, although a considerable variety of ecologies characterize the basal clades making inferences difficult. Sphagnum is an extreme aquatic, likely with a highly derived morphology. The Andreales are highly desiccationtolerant rock dwellers, and the Polytrichales show a variety of life histories ranging from small ephemerals to large perennials. There remains a need for detailed reconstructions of ecology and life history as the moss phylogeny becomes further resolved (e.g. Shaw et al., 2005). In the meantime, based on out-group comparisons, we suggest that the most likely reconstruction of the primitive moss ecology would be something like the growth habit that the Funariales generally show today: modest-sized plants occupying mesic, often ephemeral sites on soil near water.

From this starting point, some lineages evolved to occupy more hydric sites, others much more xeric sites. Accompanying these transitions, a number of specialized morphological and physiological traits evolved in various lineages. Mosses are not a collection of evolutionary dead ends; on the contrary, they are as highly evolved in their ways as the tracheophytes are in 
theirs. The ways of mosses are different, not inferior, hence the aversion of moss biologists to hearing their organisms called 'lower plants'!

\subsection{Evolution and ecology of the mosses}

Mishler (2001) postulated that moss biology is different from that of tracheophytes in most ways, representing alternative strategies to being a land plant that are almost as different from each other as one might expect to see developing on different planets (suggesting that 'mosses are from Mars, vascular plants are from Venus'). We would like to elaborate here on some of the most important factors of this unique biology of mosses. When comparing the model moss $P$. patens with tracheophytes, it is important to understand both its similarities and differences with its larger cousins.

\subsubsection{Desiccation tolerance and poikilohydry}

As plants transitioned from an aqueous environment to the land, an event that is thought to have occurred from fresh water habitats rather than marine (Mishler and Churchill, 1985), the ability to survive periods of water depletion both from the plant and its surroundings became a critical aspect of their existence. All plants, and for that matter all organisms, require water in order to sustain metabolic activity and survive. Because of the strong selective pressure of dealing with desiccation, Oliver et al. $(2000,2005)$ postulated that early land plants had to have acquired desiccation tolerance as a heritable trait in order to survive in terrestrial environments. The need for desiccation tolerance was particularly critical for survival on land given that, in all likelihood, the water relations of early land plants were poikilohydric in nature because of their simple, generally one cell thick, architecture, similar to what we see in many modern-day bryophytes.

Poikilohydry is defined as the direct equilibration of a plant's water content to the water content of the environment (generally the air) that surrounds it, and is probably the most important single difference between bryophyte and tracheophyte biology - the latter being termed homeohydric or endohydric because of their ability to internalize water relationships by means of thick cuticles, stomates, efficient conducting tissues and roots. When free water is available, a poikilohydric plant is hydrated and can be physiologically active; when free water is lost, the plant, because it does not have the mechanisms in place to retain water, will equilibrate to the water content of the surrounding air, which is generally low, and thus desiccate. Bryophytes, including $P$. patens, have retained the original life cycle for land plants, with a dominant haploid gametophytic stage where the plants are a few layers thick. This architecture dictated that bryophytes are by nature poikilohydric. Other important biological correlates of this life cycle and poikilohydry are discussed below. 
Poikilohydry is not necessarily combined with desiccation tolerance. Some aquatic bryophytes are poikilohydric yet not desiccation tolerant, while a few phylogenetically scattered tracheophytes are desiccation tolerant yet endohydric. However, poikilohydric plants must be desiccation tolerant in all but hydric situations in order to survive.

In order for growth to occur, plants must fix carbon for both structural development and energy storage. Carbon fixation requires that plant cells remain fully hydrated, which in dry environments (i.e. most terrestrial habitats) presents a challenge. In order to access $\mathrm{CO}_{2}$ from the air, plants must expose themselves to a drying atmosphere with the associated water loss. The larger and more complex tracheophytes have evolved mechanisms to extract water from the environment, principally from the soil via roots, and transport it through the vegetative tissue via tracheids to the organs (leaves) that serve as the site of $\mathrm{CO}_{2}$ uptake and fixation. Leaves of tracheophytes control the balance between $\mathrm{CO}_{2}$ uptake and water loss by employing a waxy cuticle that severely limits evaporation from their surfaces and by utilizing a system of controlled openings into the leaf structure (stomates), for passage of gases and water vapor to and from the photosynthetically active cells. The loss of water vapor, that is, transpiration, from the leaves is also necessary, regardless of the risk of dehydration, for survival, as it is the only mechanism the plant has to regulate the temperature of the leaves (by cooling) that would otherwise overheat from the incoming radiant energy from the sun that they have evolved to capture and use. Bryophytes do not have the structural capacity to efficiently transport water internally from the soil to the site of photosynthesis in the leaves, and although some do have thin waxy cuticles to slow evaporation, most rely on the maintenance of a surface film of free water to remain hydrated. Several features of mosses, the most important of which are the establishment of a colonial lifestyle in clumps and the sacrificing of certain cells that act to form external capillaries for movement of water to the active leaf cells, serve to maintain a surface film of water. Consequently, gaseous exchange and carbon fixation in these plants occur primarily within an aqueous environment and thus these plants only grow when free surface water is available. To accommodate this, bryophytes either live in habitats that are essentially always wet or they have to suffer and survive desiccation in the same way as the primitive plants that first invaded the dry land.

While most mosses can tolerate some dehydration, not all are desiccation tolerant. Desiccation tolerance is defined as the ability to survive equilibration to the water content of dry air, and means that the plant can survive, repair and recover from the damage incurred during desiccation or during the inrush of water that occurs when the plant is rehydrated. Note that desiccation tolerance is not at all the same (either at the phenotypic or genotypic level) as 'water stress tolerance' or more correctly 'dehydration tolerance', defined as the ability to survive low water contents.

The difference between desiccation tolerance and dehydration tolerance is illustrated by the desiccation-tolerant moss Tortula ruralis. When placed 
in an atmosphere that is at a constant $50 \%$ relative humidity and at $20^{\circ} \mathrm{C}$, T. ruralis will equilibrate to the water content of the air approximately $4-5 \mathrm{~h}$ after the removal of free water from the surface of the leaves (M.J. Oliver, unpublished). T. ruralis, under these conditions, will dehydrate to a water content of close to $100 \mathrm{mg} \mathrm{H}_{2} \mathrm{O} \mathrm{g}^{-1}$ dry mass and a water potential of $-100 \mathrm{MPa}$ (Gaff, 1997), and yet still be able to recover when rewetted. By contrast, although $P$. patens does not tolerate desiccation under normal circumstances and is thus considered a desiccation-sensitive plant. P. patens is capable of surviving severe dehydration, reported as the loss of $95 \%$ of its fresh weight, for short periods of time (Frank et al., 2005).

The ability to tolerate severe dehydration, while not tolerating desiccation, appears to be common in mesic bryophytes. Interestingly, for the few mesic species that have been investigated, equilibration to water potentials of $-100 \mathrm{MPa}$ and lower (which would constitute true desiccation tolerance) can be achieved by the application of exogenous abscisic acid (ABA) (Alpert and Oliver, 2002; Oliver, 2007). For example, protonemal cultures of the mesic moss Funaria hygrometrica can survive dehydration to approximately $-70 \mathrm{MPa}$ if water loss is slow but cannot survive if water loss is rapid (Werner et al., 1991). Pre-treatment of the protonemata of this moss with ABA enables them to survive rapid desiccation rates and this alteration in phenotype is associated with the accumulation of specific proteins (Bopp and Werner, 1993), some of which resemble the dehydrins known from desiccation-tolerant species such as T. ruralis (Velten and Oliver, 2001). ABA treatment of the moss Atrichum androgynum results in a desiccation-tolerant phenotype and a significant reduction in dehydration-induced cellular leakage, indicative of an increase in membrane stability (Beckett, 1999). The effect of ABA on the membrane integrity of $A$. androgynum has been investigated further, and although there was no specific effect, ABA treatment does reduce the overall level of dehydration-induced alteration in lipid membrane components (Guschina et al., 2002). The implication of these studies is that ABA induces transcriptional changes that direct the synthesis of protective cellular components that increase the ability of these plants to survive severe dehydration; dehydrins appear to be a major aspect of this response (see Saavedra et al., 2006; for more details, see Chapter 11). Exploration of the evolutionary, ecological and genomic links between dehydration tolerance (water-deficit stress) and desiccation tolerance presents an exciting area for future studies.

The link between water and carbon fixation, as it relates to growth, can be best summarized by the Integrated Water-driven Carbon Budget (IWCB) model, as described in Figure 1.3. This model seeks to describe the relationship between water and carbon fixation for all plants, regardless of their mechanism for dealing with life on land and maintaining growth under conditions of limiting water resources. It also offers a way to quantify this relationship and establish a means by which species can be directly compared with regards to their ability to tolerate dehydration. The IWCB model describes the 


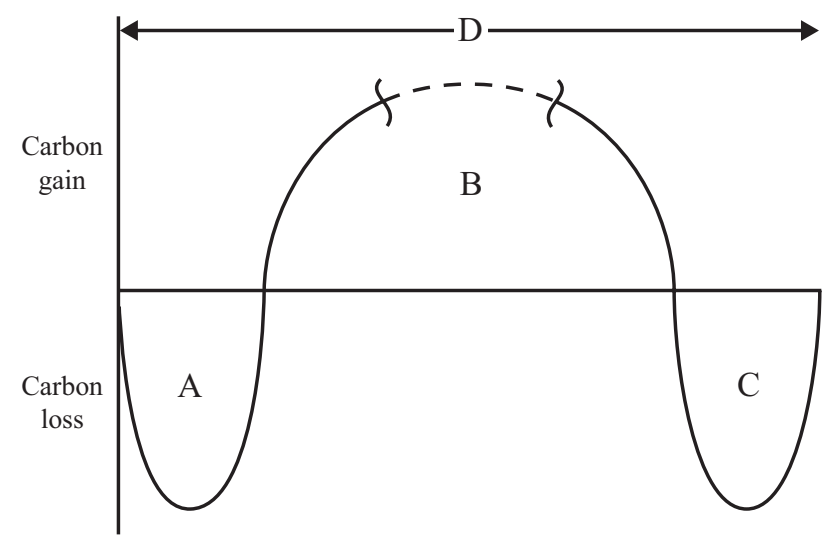

Figure 1.3 Integrated water-driven carbon budget (IWCB) model. This diagram represents the events following dryness until the next period of dryness, that is, one dry-wet-dry cycle (of time D) in the life of a plant. The dried plant is assumed to have a zero carbon balance as metabolism has ceased. When water is first added, there is an initial phase of net carbon loss (A). Then, as the plant repairs itself and begins photosynthesis, the compensation point is eventually reached, and the plant enters a phase of net carbon gain (B). Finally, as the plant begins to dry the curve starts to turn down since photosynthesis is limited, and the plant enters a second phase of net carbon loss (C), eventually drying completely. The net carbon gain for a given amount of water is the area under the curve in B minus the area under the curve in $A+C$. Plants can vary in many ways under this model; shapes of the curves for different plants can differ in each of these areas.

amount of carbon fixed for a given amount of water during one dry-wet-dry cycle within the lifetime of a plant. The traditional term 'water use efficiency' (WUE) only applies to the ability of a plant to fix a certain amount of carbon per unit water in phase B. Thus, the IWCB model is a broader measure of the overall water relationships of a plant than WUE. Although this model applies to the IWCB for all plants, it more completely describes the relationship between net carbon fixation and water use for poikilohydric plants, and in particular those plants that are desiccation tolerant. Endohydric plants (e.g. the majority of the angiosperms), those that are desiccation sensitive and have the capability to maintain the hydrated condition, spend their whole photosynthetic lifetime in phase B of the IWCB model. Desiccation-tolerant plants, whether poikilohydric or not, will experience many of the dry-wet-dry cycles within their normal life spans, many years in the case of most bryophytes. P. patens is, however, capable of surviving severe dehydration, reported as the loss of $95 \%$ of its fresh weight, for short periods of time (Frank et al., 2005). If this occurs under natural conditions, perhaps allowing the plant to survive brief periods of drying, the IWCB would reflect brief periods in both A and C stages of the dry-wet-dry cycle. 


\subsubsection{Reproductive biology}

All bryophytes need free water for sexual reproduction as well as for metabolism. This is another residual feature remaining from the early land plants, a constraint imposed by swimming sperm inherited from their aquatic ancestors among the green algae. Swimming gametes have relatively short dispersal distances as compared to pollen grains, which presumably lead to frequent inbreeding in monoicous species (i.e. those bearing male and female gametangia on the same gametophyte) and potentially decreased sporophyte production in dioicous species (i.e. those bearing male and female gametangia on different gametophytes).

Due to the difficulty of achieving fertilization in the terrestrial environment, many bryophytes seldom reproduce sexually and some may even have evolutionarily lost functional sexuality. For example, a significant proportion (about 9\%; Lane, 1985) of moss species in eastern North America have never been observed to produce a sporophyte. There is presumably a heavy reliance on asexual reproduction in many species; many types of specialized propagules are found in different species (Newton and Mishler, 1994), and all organs of a moss can serve as propagules by regenerating protonemata. Since mosses that are dioicous have a special problem with effecting fertilization, they appear to have more frequently evolved specialized asexual propagules (Mishler, 1990). Even in monoicous species such as P. patens, which frequently produce sporophytes in nature, it is important to keep in mind that the great majority of these sporophytes likely result from self-fertilization. In a haploid, selfing has very different implications from those in diploids where the result is complete homozygosity. Thus, spores from selfed sporophytes are genetically asexual propagules, perhaps explaining why monoicous taxa seem to have evolved specialized asexual propagules less often than dioicous taxa (Mishler, 1990).

\subsubsection{Population biology}

Many mosses are predominantly clonal because of several fundamental aspects of their biology. First, uniquely among land plants, they develop many stems from a single radiating protonemal system (derived from a single spore). Second, their various modes of branching, and persistence of older branches, mean that they expand clonally in a growth form characteristic of a species. Third, rampant asexual reproduction means that clones spread near and far. Newton and Mishler (1994) pointed out that the available evidence suggests that most new establishment events, especially close to the parent plant in crowded situations, are due to asexual propagules. They suggested that the ecological role of spores might be in longer distance dispersal and rare colonization of bare habitats.

Unlike tracheophytes, the green, vegetative part of the life cycle in bryophytes is haploid. Haploid dominance in the life cycle has major 
implications. As described above, self-fertilization results in spores that are genetically similar to asexual propagules. Furthermore, without the genetic benefits of dominance, genes acting in the gametophyte are presumably subject to relatively severe selection. Haploidy plus clonal growth might suggest that levels of genetic variation within populations should be low. Some studies suggest this is the case (e.g. Shaw et al., 2002); however, other studies show significant variation within populations (McDaniel and Shaw, 2005). Since bryophytes grow from an apical cell, somatic mutation allows genetic variation even within clones (Klekowski, 1988), and indeed appreciable levels of genetic variation have been shown within and among populations of asexual species (i.e. those in which sporophytes have not been seen in nature).

Bryophytes tend to have very high amounts of morphological and physiological plasticity. Interestingly, while there appears to be considerable genetic variation within many bryophyte species, few examples of ecotypic differentiation (i.e. genetically based, locally adaptive variation) have been found in mosses to date, although more studies are needed (see Chapter 2). For example, the cosmopolitan moss Bryum argenteum has very similar physiological tolerances in the tropics as in the Antarctic (Longton, 1981), indicating that phenotypic plasticity can allow occupation of a broad range of environments. Studies of physiological variation within and among species of mosses present an exciting area for future research. Many genotypes of $P$. patens have been gathered from around the world and are now in culture in Freiburg (see http://www.cosmoss.org/ecomap.content\#); these provide a rich source of material for such studies.

\subsubsection{Ecology}

Poikilohydry has a number of ecological correlates in addition to those discussed above. One is acute sensitivity to the chemical environment surrounding the plant, unlike anything seen in a rooted plant which brings its water to vegetative cells from below in the soil. Since water is crossing directly into the vegetative cells of mosses across the cell membrane, chemical entities in that water tend to enter the cell as well, and vice versa as the cell dries out and loses water into the immediate environment. Thus, mosses are quite sensitive to ions in the environment and are good ecological indicators of their substrate (e.g. pH, amount of calcium in a rock, or bark chemistry of a tree). Mosses are also sensitive to a range of pollutants, showing the impact of air pollution before endohydric plants. For example, the sulfur content of mosses is an exact indicator of $\mathrm{SO}_{2}$ pollution levels in the air and thus can be used to monitor pollution from factories (de Caritat et al., 1997). Conversely, as the moss dries out, it loses cellular components to the environment, and this is thought to facilitate growth of symbiotic microbes, of which mosses appear to have many.

The combination of clonal growth with poikilohydry and external water holding and conduction means that we can best view most mosses as 
essentially social organisms. The moss clump is the unit that interacts with the environment; the plants in a clump are subject to natural selection as a group. The moss clump is thus a 'super-organism' somewhat like a beehive. Intimate contact of each vegetative cell with the environment, due to poikilohydry, lends itself to interplant chemical communication via pheromones. Such communication has been suggested to explain the dwarfing of males in a number of dioicous mosses, giving them a special breeding system, and to explain the complete lack of germination of spores frequently seen within mature moss clumps (Mishler and Newton, 1988).

Mosses occupy microhabitats, which has many implications. Their small size, lack of roots and poikilohydry mean that mosses are in a close relationship with only their immediate microenvironment. Over a short time scale, this means that a suitable habitat for a particular moss species is likely to exist in most macro-environments. Over geological time, mosses may thus be less influenced by climatic change than the larger tracheophytes, and linger in refugial habitats along with other small organisms.

Establishment abilities of mosses seem relatively poor. They appear to be very slow to establish in available nearby habitats, thus available substrates are not filled in most mesic and xeric environments (although they may be in some hydric environments such as bogs). The few studies that have been aimed at a demonstration of competition among dry-land mosses suggest that instead of the expected negative effects of crowding, the presence of other moss species may enhance growth because of an increased water-holding capacity (e.g. McAlister, 1995). The main selective factors seem to be the ability of propagules to survive the physical environmental stresses and establish a new clump. Herbivory appears relatively low on bryophytes. Together, these observations suggest that for many mosses, there is less selection pressure from the biotic component of the environment than from the physical component, which again is at odds with the prevailing picture of tracheophyte ecology.

\subsection{General implications for evolutionary processes in mosses}

There is no evidence that mosses have slow rates of evolution at the molecular level, or at the physiological level. However, there does appear to be relatively slow evolutionary rates in morphology in mosses; their fossil record indicates that ancient forms are very similar to modern ones, and cryptic species are present, some of them apparently quite old (McDaniel and Shaw, 2003). This may indicate that developmental constraints play an unusually important role, and suggest that studies of evolution and development in mosses would be very productive to pursue in the future.

Biogeographically, bryophytes tend to follow the same historical patterns of disjunction as tracheophytes, but at a lower taxonomic level. In other words, a moss species often has a distribution historically comparable to an 
angiosperm genus or family. There are also more molecular differences among geographically disjunct populations in moss species than one generally sees in tracheophyte species (Shaw, 2001). These recorded differences are probably the result of a difference in species concept held by those investigating the two groups. Bryologists tend to be conservative in naming species, only naming those with distinct morphological differences. Because of the relatively slow rate of morphological change, currently recognized moss species are thus undoubtedly older than many angiosperm species. It is likely that if the species level was applied in mosses at the same age level as it is in angiosperms, there might be as many species of mosses as angiosperms! Future research on mosses needs to continue probing into fine-scale phylogeny while looking more at physiological and developmental variation in order to explain their diversification. Like many other areas of moss biology, the processes influencing their evolutionary diversification seem to be quite different from the tracheophytes. Moss biologists need to declare independence from concepts imported from angiosperm and animal biology; these little plants are in a world of their own!

\section{Acknowledgments}

We thank the book editors as well as Stuart McDaniel for comments on the text, and the US National Science Foundation for support of the Green Tree of Life Project (DEB-0228729 to BDM) and the Joint Genome Institute of the US Department of Energy for funding the sequencing of the P. patens genome under their Community Sequencing Program (B.D. Mishler and R.S. Quatrano, primary proposers). We would also like to thank Steve Rice, Peter Alpert and Dave Hanson for discussion of issues of moss physiology and ecology, especially the IWCB model.

\section{References}

Alpert, P. and Oliver, M.J. (2002) Drying without dying. In: Desiccation and Survival in Plants: Drying without Dying (eds M. Black and H.W. Pritchard), pp. 3-43. CABI Publishing, Wallingford.

Beckett, R.P. (1999) Partial dehydration and ABA induce tolerance to desiccationinduced ion leakage in the moss Atrichum androgynum. South African Journal of Botany, 65, 1-6.

Bopp, M. and Werner, O. (1993) Abscisic acid and desiccation tolerance in mosses. Botanica Acta, 106, 103-106.

Cove, D.J. (2000) The moss, Physcomitrella patens. Journal of Plant Growth Regulation, 19, 275-283.

Cox, C.J., Goffinet, B., Shaw, A.J. and Boles, S.B. (2004) Phylogenetic relationships among the mosses based on heterogeneous Bayesian analysis of multiple genes from multiple genomic compartments. Systematic Botany, 29, 234-250. 
de Caritat, P., Krouse, H.R. and Hutcheon, I. (1997) Sulphur isotope composition of stream water, moss and humus from eight arctic catchments in the Kola Peninsula region (NW Russia, N Finland, NE Norway). Water, Air, and Soil Pollution, 94, 191-208.

Frank, W., Ratnadewi, D. and Reski, R. (2005) Physcomitrella is highly tolerant against drought, salt and osmotic stress. Planta, 220, 384-394.

Gaff, D.F. (1997) Mechanisms of desiccation tolerance in resurrection vascular plants. In: Mechanisms of Environmental Stress Resistance in Plants (eds A.S. Basra and R.K. Basra), pp. 43-58. Harwood Academic, London.

Goffinet, B., Shaw, J., Anderson, L.E. and Mishler, B.D. (1999) Peristome development in mosses in relation to systematics and evolution. V. Diplolepideae: Orthotrichaceae. The Bryologist 102, 581-594.

Guschina, I.A., Harwood, J.L., Smith, M. and Beckett, R.P. (2002) Abscisic acid modifies the changes in lipids brought about by water stress in the moss Atrichum androgynum. New Phytologist, 156, 255-264.

Kelch, D.G., Driskell, A. and Mishler, B.D. (2004) Inferring phylogeny using genomic characters: a case study using land plant plastomes. In: Molecular Systematics of Bryophytes (eds B. Goffinet, V. Hollowell and R. Magill), pp. 3-12. Missouri Botanical Garden Press, St. Louis, MO.

Klekowski, E.J. (1988) Mutation, Developmental Selection, and Plant Evolution. Columbia University Press, New York.

Lane, D.M. (1985) A quantitative study of The Mosses of Eastern North America. Monographs in Systematic Botany, 11, 45-50.

Longton, R.E. (1981) Inter-population variation in morphology and physiology in the cosmopolitan moss Bryum argenteum Hedw. Journal of Bryology, 11, 501.

McAlister, S. (1995) Species interactions and substrate specificity among loginhabiting bryophyte species. Ecology, 76, 2184-2195.

McDaniel, S.F. and Shaw, A.J. (2003) Phylogeographic structure and cryptic speciation in the trans-Antarctic moss Pyrrhobryum mnioides. Evolution, 57, 205-215.

McDaniel, S.F. and Shaw, A.J. (2005) Selective sweeps and intercontinental migration in the cosmopolitan moss Ceratodon purpureus (Hedw.) Brid. Molecular Ecology, 14, 1121-1132.

Mishler, B.D. (1988) Relationships between ontogeny and phylogeny, with reference to bryophytes. In: Ontogeny and Systematics (ed. C.J. Humphries), pp. 117-136. Columbia University Press, New York.

Mishler, B.D. (1990) Reproductive biology and species distinctions in the moss genus Tortula, as represented in Mexico. Systematic Botany, 15, 86-97.

Mishler, B.D. (2000) Deep phylogenetic relationships among 'plants' and their implications for classification. Taxon, 49, 661-683.

Mishler, B.D. (2001) Book review: the biology of bryophytes. American Journal of Botany, 88, 2129-2131.

Mishler, B.D. and Churchill, S.P. (1985) Transition to a land flora: phylogenetic relationships of the green algae and bryophytes. Cladistics, 1, 305-328.

Mishler, B.D. and Newton, A.E. (1988) Influences of mature plants and desiccation on germination of spores and gametophytic fragments of Tortula. Journal of Bryology, 15, 327-342.

Newton, A.E., Cox, C.J., Duckett, J.G. et al. (2000) Evolution of the major moss lineages: phylogenetic analyses based on multiple gene sequences and morphology. The Bryologist, 103, 187-211. 
Newton, A.E. and Mishler, B.D. (1994) The evolutionary significance of asexual reproduction in mosses. Journal of the Hattori Botanical Laboratory, 76, 127-145.

Oliver, M.J. (2007) Lessons on dehydration tolerance from desiccation-tolerant plants. In: Plant Desiccation Tolerance (eds M. Jenks and A.J. Wood), pp. 11-42. Blackwell, Ames, IA.

Oliver, M.J., Tuba, Z. and Mishler, B.D. (2000) Evolution of desiccation tolerance in land plants. Plant Ecology, 151, 85-100.

Oliver, M.J., Velten, J. and Mishler, B.D. (2005) Desiccation tolerance in bryophytes: a reflection of the primitive strategy for plant survival in dehydrating habitats? Integrated and Comparative Biology, 45, 788-799.

Pryer, K.M., Schneider, H., Smith, A.R. et al. (2001) Horsetails and ferns are a monophyletic group and the closest living relatives to seed plants. Nature, 409, 618-622.

Qiu, Y.-L., Li, L., Wang, B. et al. (2006) The deepest divergences in land plants inferred from phylogenomic evidence. Proceedings of the National Academy of Sciences of the United States of America, 103, 15511-15516.

Rensing, S.A., Lang, D., Zimmer, A.D. et al. (2008) The Physcomitrella genome reveals evolutionary insights into the conquest of land by plants. Science, 319, 64-69.

Reski, R. (1998) Development, genetics and molecular biology of mosses. Botanica Acta, 111, 1.

Reski, R. (1999) Molecular genetics of Physcomitrella. Planta, 208, 301.

Saavedra, L., Svensson, J., Carballo, V., Izmendi, D., Wellin, B. and Vidal, S. (2006) A dehydrin gene in Physcomitrella patens is required for salt and osmotic stress tolerance. The Plant Journal, 45, 237-249.

Shaw, A.J. (2001) Biogeographic patterns and cryptic speciation in bryophytes. Journal of Biogeography, 28, 253-261.

Shaw, A.J., Cox, C.J. and Goffinet, B. (2005) Global patterns of moss diversity: taxonomic and molecular inferences. Taxon, 54, 337-352.

Shaw, A.J., McDaniel, S.F., Werner, O. and Ros, R.M. (2002) Phylogeography and phylodemography. The Bryologist, 105, 373-383.

Smith, D.K. and Davison, P.G. (1993) Antheridia and sporophytes in Takakia ceratophylla (Mitt.) Grolle: evidence for reclassification among the mosses. Journal of the Hattori Botanical Laboratory, 73, 263-271.

Velten, J. and Oliver, M.J. (2001) Tr288: a rehydrin with a dehydrin twist. Plant Molecular Biology, 45, 713-722.

Werner, O., Espin, R.M.R., Bopp, M. and Atzorn, R. (1991) Abscisic-acid-induced drought tolerance in Funaria hygrometrica Hedw. Planta, 186, 99-103. 